OPEN ACCESS

Edited by:

Jean-Philippe Lambert, Laval University, Canada

Reviewed by:

Jeffrey Fillingham,

Ryerson University, Canada Craig Peterson,

University of Massachusetts Medical

School, United States

*Correspondence:

Lóránt Székvölgyi lorantsz@med.unideb.hu

tORCID:

Lóránt Székvölgyi

orcid.org/0000-0002-7529-0319

Specialty section:

This article was submitted to Epigenomics and Epigenetics,

a section of the journal

Frontiers in Cell and Developmental

Biology

Received: 25 October 2019 Accepted: 12 December 2019

Published: 10 January 2020

Citation:

Karányi Z, Hornyák L and Székvölgyi L (2020) Histone H3 Lysine

56 Acetylation Is Required for Formation of Normal Levels of Meiotic DNA Breaks in S. cerevisiae.

Front. Cell Dev. Biol. 7:364. doi: 10.3389/fcell.2019.00364

\section{Histone H3 Lysine 56 Acetylation Is Required for Formation of Normal Levels of Meiotic DNA Breaks in S. cerevisiae}

\author{
Zsolt Karányi ${ }^{1,2}$, Lilla Hornyák ${ }^{1}$ and Lóránt Székvölgyi ${ }^{1 *+}$ \\ ${ }^{1}$ MTA-DE Momentum Genome Architecture and Recombination Research Group, Department of Biochemistry \\ and Molecular Biology, Faculty of Medicine, University of Debrecen, Debrecen, Hungary, ${ }^{2}$ Department of Internal Medicine, \\ Faculty of Medicine, University of Debrecen, Debrecen, Hungary
}

Meiotic recombination is initiated by Spo11-catalyzed DNA double-strand breaks (DSBs) that are promoted by histone modifications and histone modifying enzymes. Herein we investigated the role of histone $\mathrm{H} 3$ lysine 56 acetylation (H3K56ac) located near the entry/exit points of the DNA in the globular $\mathrm{H} 3$ domain. We generated a series of mutant cells (asf1 $\Delta, r t t 109 \Delta, h s t 3 / 4 \Delta$, and H3K56A) in which the endogenous level of H3K56ac was manipulated and tracked during meiotic growth. We show that complete loss or increased abundance of H3K56ac in these mutants allows timely entry into meiosis and sporulation and does not impair S phase progression, first and second

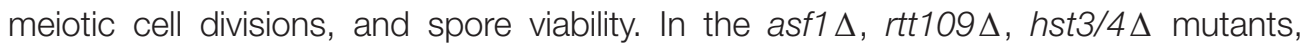
DSBs and crossovers form normal levels with a short (60-min) delay at the HIS4-LEU2 artificial recombination hotspot, however, DSB formation shows a $\sim$ threefold decrease in the H3K56A mutant at the natural BUD23-ARE1 hotspot. The latter DSB phenotype, showing significant DSB reduction in the H3K56A mutant, was also observed at DSB sites using genome-wide mapping of Rfa1-coated single-stranded DNA flanking DSBs (RPA ChIP). Parallel mapping of H3K56-acetylated histones in wild type cells revealed strong depletion of the H3K56ac ChIP signal over Spo11-oligo DSBs, albeit most H3K56-acetylated histones were enriched adjacent to the identified RPA ChIP binding sites. Taken together, these associations demonstrate a prominent role of H3 lysine 56 acetylation in the formation of DNA breaks within recombination hotspot regions.

\section{Keywords: recombination, DNA break, meiosis, histone modification, H3K56 acetylation}

\section{INTRODUCTION}

Meiosis is a cellular differentiation process which is accompanied by high levels of recombination between the homologous chromosomes, initiated by DNA double-strand breaks (DSBs) catalyzed by Spo11 and accessory factors (Keeney et al., 1997; Székvölgyi and Nicolas, 2010; Székvölgyi et al., 2015). In S. cerevisiae, meiotic DSBs are controlled by the elements of chromatin structure. This involves a complex interplay between DNA sequence composition, local chromatin status, nucleosome occupancy, and transcription factor binding (Pan et al., 2011). Among these factors, histone modifications represent an important layer which has only recently been explored in detail. The most well-characterized histone modification is H3K4me3 that appears to be essential for recombination sites in most organisms. In the current model, H3K4me3 is deposited by Set1C 
and becomes recognized by Spp1 (the PHD finger subunit of Set1C), which leads to tethering of DSB sites to the chromosome axis that undergo Spo11-mediated cleavage (Acquaviva et al., 2013; Sommermeyer et al., 2013; Karányi et al., 2018). A similar mechanism has been proposed in mammals involving the meiosis-specific H3K4 methylase Prdm9 (Baudat et al., 2010; Parpanov et al., 2010), however, CXXC1 (the yeast ortholog of Spp1) is apparently not essential for the association of H3K4 tri-methylated recombination sites with the DSB machinery (Tian et al., 2018).

The widely localized H3K4me3 mark has the virtue of initiating recombination at numerous places in the genome, however, other histone modifications are also needed to keep recombination flexible for the diversity of recombinant haplotypes (Szekvolgyi and Nicolas, 2010). These "alternative" pathways remain to be clarified to better understand the plasticity of crossover patterning. Most chemical modifications are concentrated at the N-termini of histones and are not expected to alter the structure of nucleosomes (White et al., 2001; Biswas et al., 2011). However, modifications of histone core domains can directly change nucleosome structure, which is wellestablished biochemically (Luger et al., 1997; Biswas et al., 2011) but its functional relevance is less understood.

In the current study, we focused on the role of histone $\mathrm{H} 3$ lysine 56 acetylation located near the entry/exit points of the DNA in the globular H3 domain, predicted to destabilize the histone/DNA contact (Buning and Van Noort, 2010; Simon et al., 2011). H3K56ac is a transient chromatin signal showing rapid turnover and is closely linked to DNA replication and histone eviction during transcription (Rufiange et al., 2007; Watanabe et al., 2013). Functional studies in mitotically proliferating yeast cells revealed that H3K56ac enables the assembly and disassembly of nucleosomes during DNA synthesis and upon transcriptional activation. Furthermore, the histone chaperone Asf1 [carrying the H3K56-specific acetyltransferase Rtt109 (Abshiru et al., 2013)] and the histone residue H3K56 were found to be necessary for meiotic $S$ phase progression and for the development of reproductive capacity in yeast and mouse models (Recht et al., 2006; Govin et al., 2010; Messiaen et al., 2016). However, the mechanism of action of H3K56 acetylation has not been fully elucidated and remains to be clarified. Therefore, we applied a functional approach in meiotic $S$ cerevisiae cells to modify the natural levels of $\mathrm{H} 3 \mathrm{~K} 56 \mathrm{ac}$ to study the biochemical phases of meiosis. Our results demonstrate that H3K56ac is necessary for formation of normal levels of DSBs within recombination hotspot regions.

\section{METHODS}

All methods are available as Supplementary Material.

\section{RESULTS}

We generated mutant cells in which the endogenous level of H3K56ac was modified and tracked during meiotic growth
(Figure 1A). In wild-type cells, the level of $\mathrm{H} 3 \mathrm{~K} 56 \mathrm{ac}$ reached a peak during DSB formation (at $\sim 4-5 \mathrm{~h}$ in SPM) and then rapidly dropped, similar to earlier western blot results (Recht et al., 2006). The H3K56ac signal disappeared almost completely from asfi $1 \Delta$ and $r t t 109 \Delta$ deletion mutants during the meiotic time course ( $\sim$ fourfold reduction compared to wild type), in agreement with the crucial role of Asf1 and Rtt109 in the deposition of this epigenetic tag (Tsubota et al., 2007). The K56ac signal increased and remained high in the hst $3 / 4 \Delta$ doublemutant that prevents deacetylation of H3K56 (Celic et al., 2006). In mitotic growth conditions, we could not detect an H3K56 acetylation signal, except for the $h s t 3 / 4 \Delta$ mutant (Figure 1A), in which $\mathrm{H} 3 \mathrm{~K} 56 \mathrm{ac}$ persists throughout the cell cycle (Celic et al., 2006). This is in accordance with the transient nature of H3K56 acetylation in asynchronously proliferating mitotic cells, mainly associated with newly replicated chromatin regions (Simoneau et al., 2015).

The correlation between H3K56ac dynamics and the phase of meiotic DSBs prompted us to analyze the progress of $S$ phase, DSB formation, sporulation efficiency, and spore viability in cells with various $\mathrm{H} 3 \mathrm{~K} 56 \mathrm{ac}$ levels. FACS analysis revealed a 30-60 min delay in G1/S phase progression in mutant cells, however, all mutants reached the G2/M phase within $6 \mathrm{~h}$ in SPM (Figures 1B,C). We then performed a meiotic time course up to $20 \mathrm{~h}$. The results reported in Figures 1D,E show that sporulation efficiencies and spore viabilities do not differ between mutant and wild-type cells (less than 1.5-fold change was observed in the number of tetrads and viable spores). We conclude that absence or elevated levels of H3K56ac is dispensable for meiotic S phase progression, MI/MII cell divisions, and spore viability.

We next analyzed whether DSBs were affected in mutants (with $\mathrm{Sae}^{+} / \mathrm{Rad} 50^{+}$background). DSB levels were monitored at the HIS4-LEU2 hotspot (Xu and Kleckner, 1995) using Southern blot hybridization (Figure 2A). All mutants produced comparable DSB levels relative to wild-type (between 6 and $8 \%$ based on the global maxima of the curves), with a $\sim 60$ min delay in asf $1 \Delta$ and $h s t 3 / 4 \Delta$ mutants (Figure 2C).

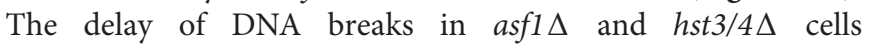
suggests that DSBs appear and disappear with a slightly modified kinetics in these mutants. Maximal DSB reduction

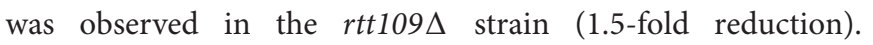
Importantly, meiotic DSBs detected in the mutants were highly recombinogenic (R1/R2 recombinants are highlighted in Figure 2B) forming wild-type levels of crossovers $(\sim 10$ $11 \%$, Figure 2C). These results suggest that DSBs detected at HIS4-LEU2 were properly processed to form mature recombination products.

To obtain mechanistic insights whether the modifiable H3K56 residue influences DSB formation, we constructed a plasmid shuffle system that allows the expression of wildtype H3K56 (H3 ctrl) and unmodifiable H3K56A as the only source of histone $\mathrm{H3}$ (Figure 3A). Like wild-type cells, H3K56A mutants progressed synchronously through the meiotic S phase (Figure 3B) and showed normal transcriptome dynamics with no difference in gene expression during meiosis (Figure 3C and Supplementary Table S1). "Core" 


\section{A}

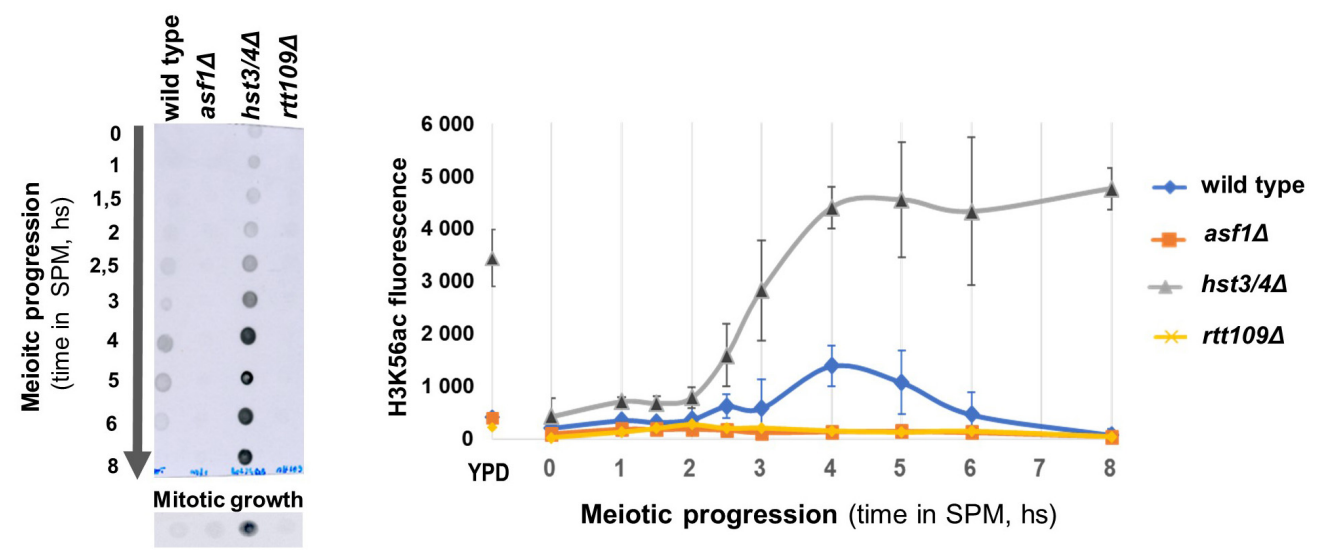

B

Meiotic progression (time in SPM, hs)
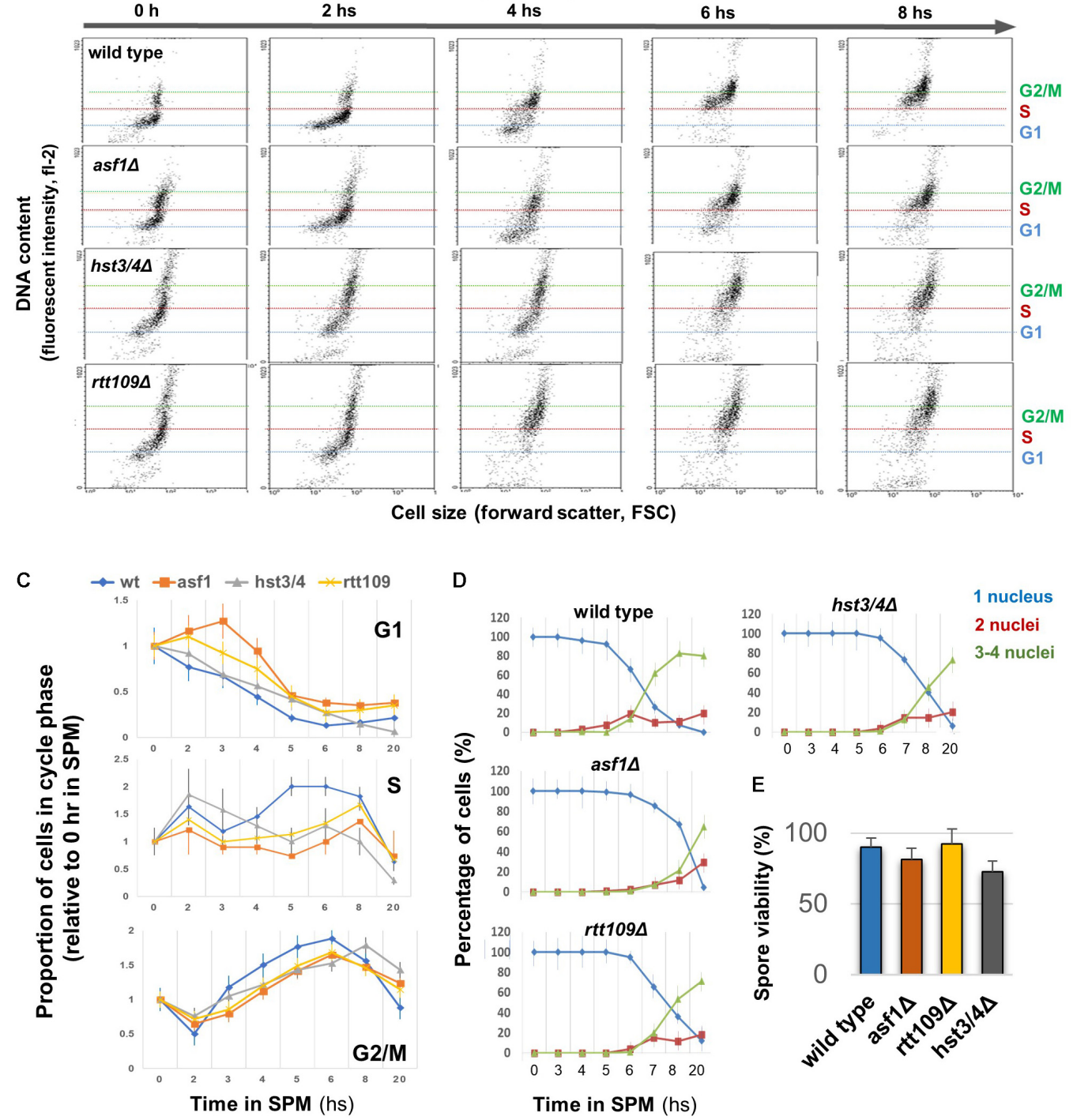

D
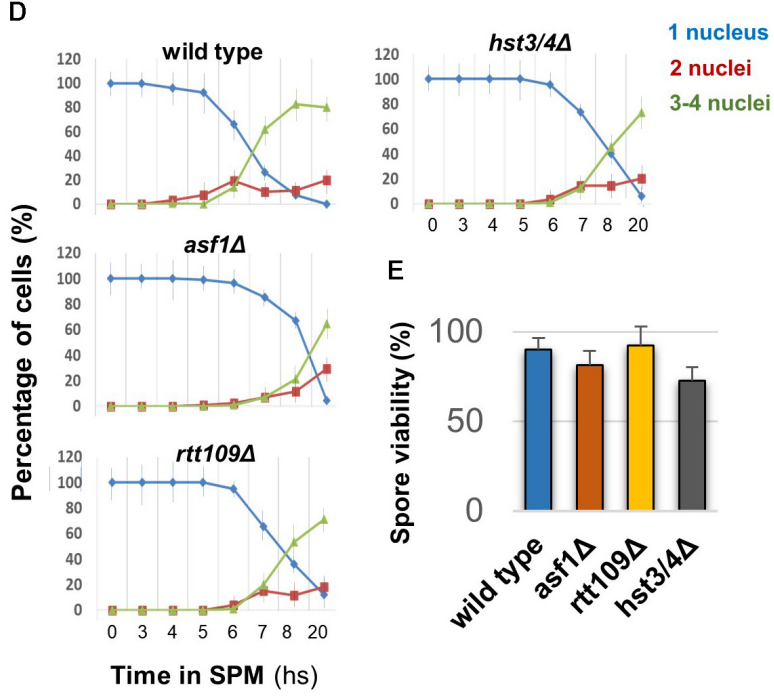

FIGURE 1 | Different H3K56ac levels allow normal meiotic progression and sporulation. (A) Left: Representative dot blot showing H3K56ac levels in wild type, asf1 $\Delta, r t t 109 \Delta$, and $h s t 3 / 4 \Delta$ cells. Right: Quantification of H3K56ac levels. YPD: mitotic growth. (B) Representative FACS profile of sporulating strains, showing DNA content in terms of cell size. G1, S, G2/M phases are indicated. (C) Proportion of cells in G1, S, and G2/M based on meiotic FACS profiles. Data were normalized to $0 \mathrm{~h}$ in SPM. (D) Sporulation efficiency. Nuclei were stained with DAPI and cells were scored for nucleus count. (E) Spore viability (50 tetrads per strain were counted). Panels (A,C-E) show the mean of three independent replicas. Error bars: SEM. 


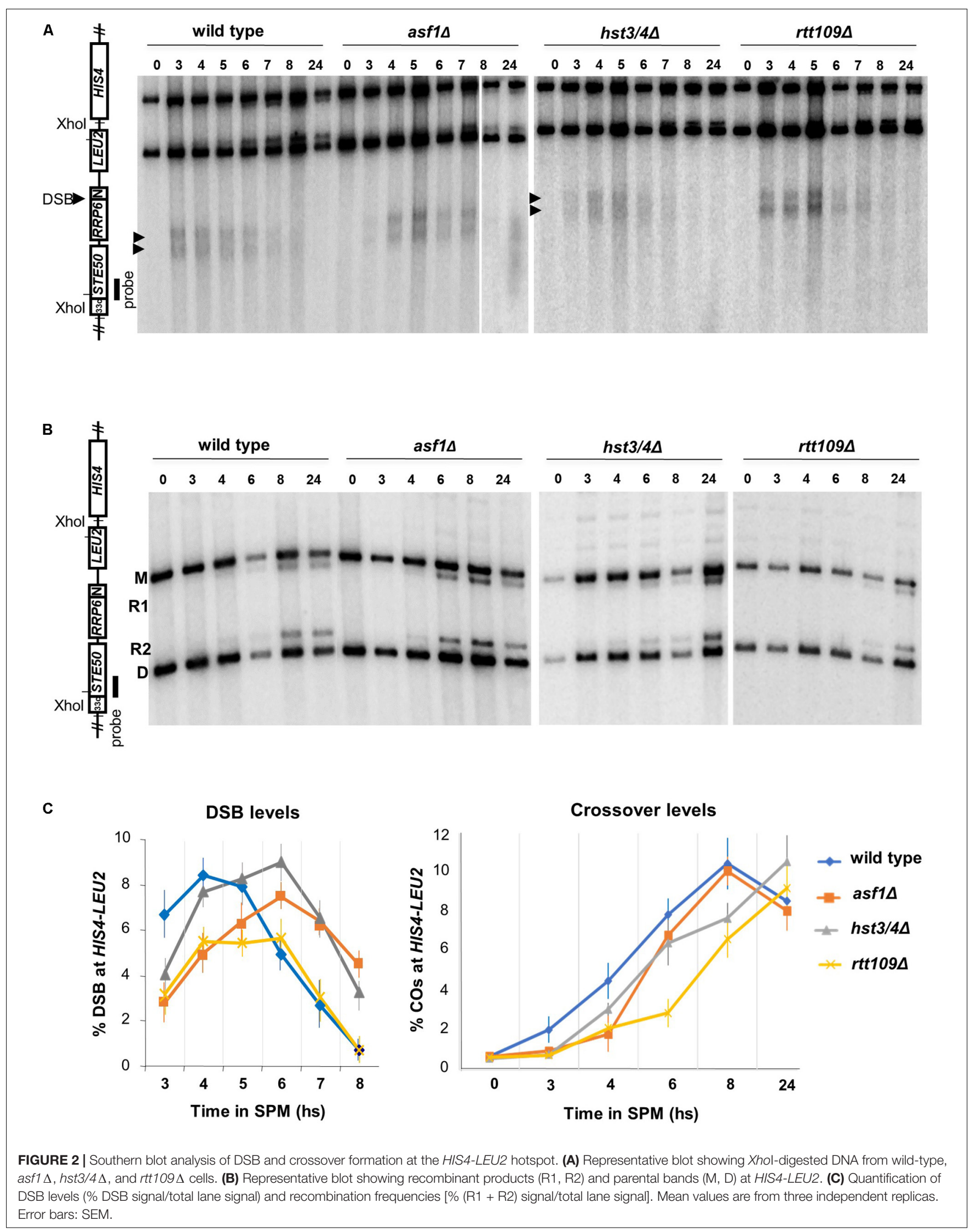


A

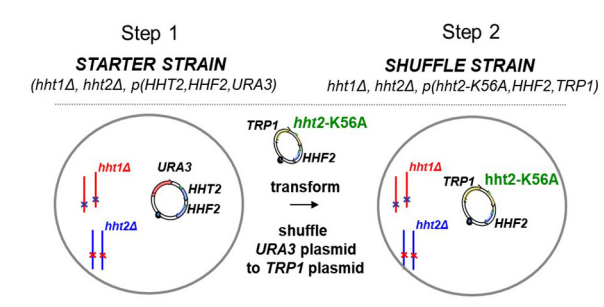

C
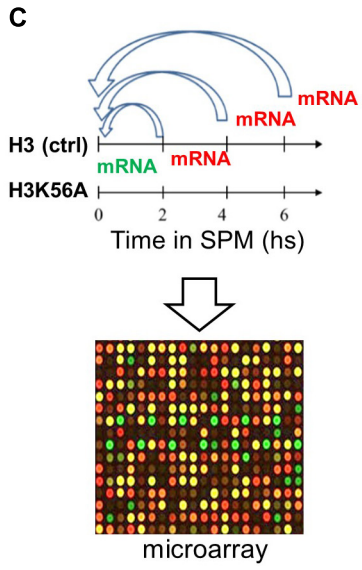
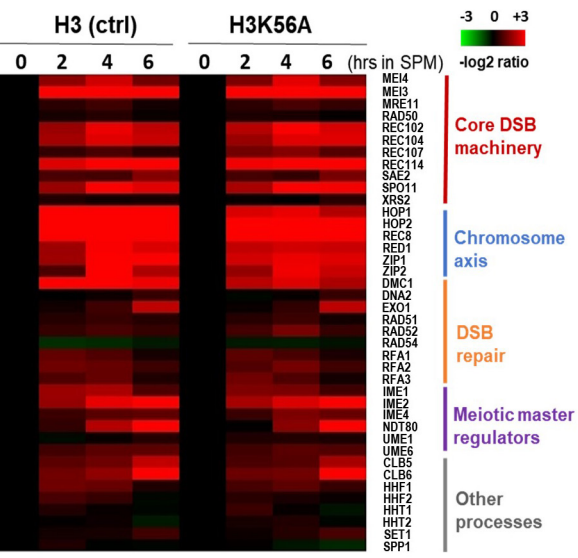

D
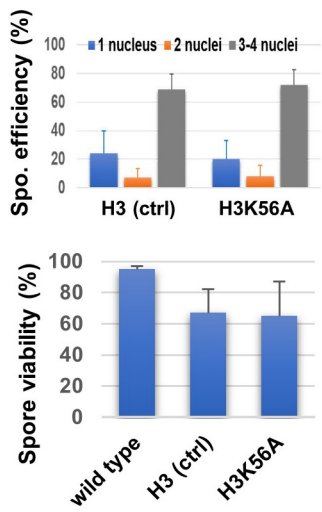

E
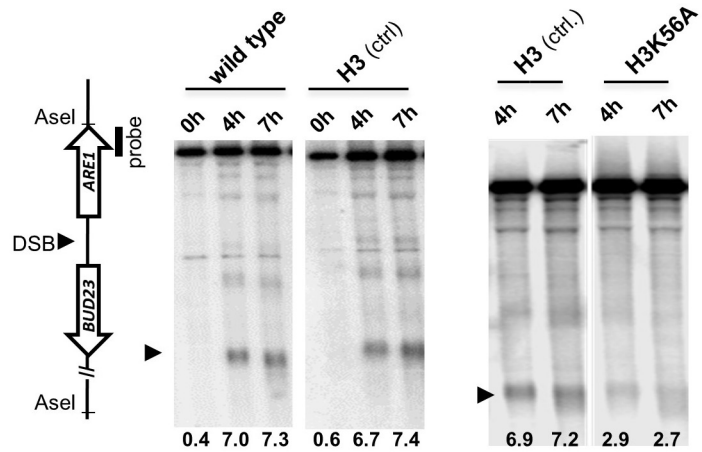

F
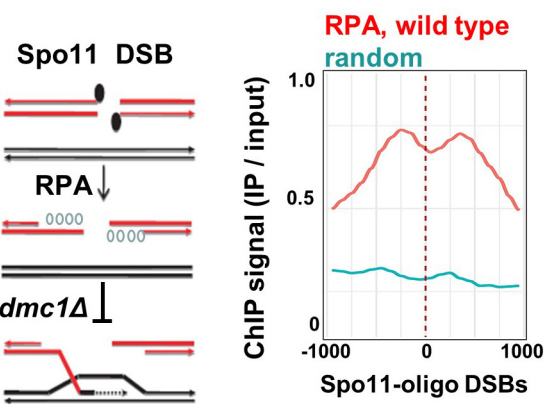

G

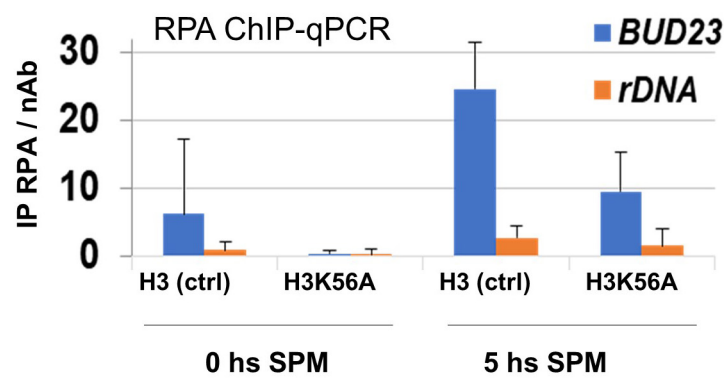

H

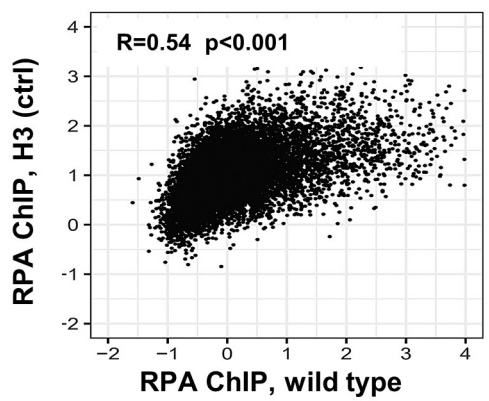

FIGURE 3 | Sporulation and meiotic DSB formation in a H3K56A mutant. (A) H3K56A cells were constructed by plasmid shuffling. (B) $\mathrm{S}$ phase progression in H3K56A and H3 (ctrl) cells measured by FACS. (C) Meiotic transcriptome dynamics in H3 (ctrl) and H3K56A strains. Left: Relative mRNA levels as measured by transcriptome microarrays at 2, 4, $6 \mathrm{~h}$ in SPM compared to $0 \mathrm{~h}$ in SPM. Right: Heatmap highlighting a selection of "core" genes that govern the process meiosis. The warmer the color, the greater the degree of induction. Full details are provided in Supplementary Table S1. (D) Sporulation efficiency and spore viability of H3K56A, H3 (ctrl), and wild-type strains. Error bars: SEM. (E) DSBs within BUD23-ARE1. Representative blot shows Asel-digested gDNA from wild type, H3 (ctrl) and H3K56A cells. DSB levels (below the blots): \% DSB signal/total lane signal. (F) Left: Outline of the RPA ChIP approach, showing resected ssDNAs covered by RPA, which accumulates in dmc1 $\Delta$ cells. Right: RPA enrichment over Spo11-oligo DSB sites and random sites in wild-type SK1 cells. Wild-type RPA data are from Borde et al. (2009). (G) ChIP-qPCR analysis of RPA enrichment at 0 and $5 \mathrm{~h}$ in SPM in the BUD23 hotspot and the rDNA negative control site (known to lack DSBs). Error bars: SEM. (H) Correlation of RPA ChIP profiles in H3 (ctrl) and wild-type cells. R: Pearson correlation coefficient. 


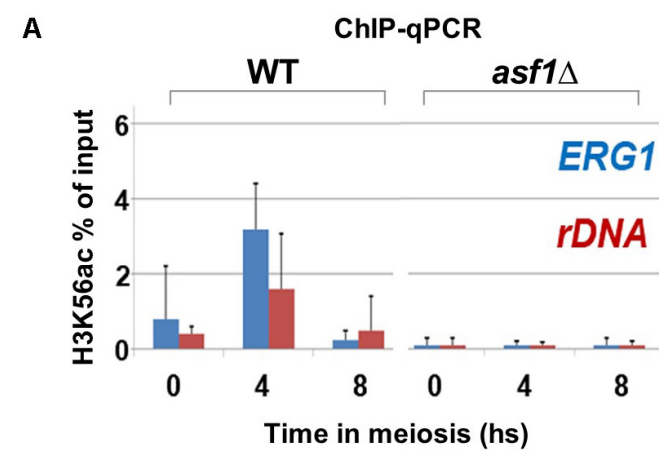

C

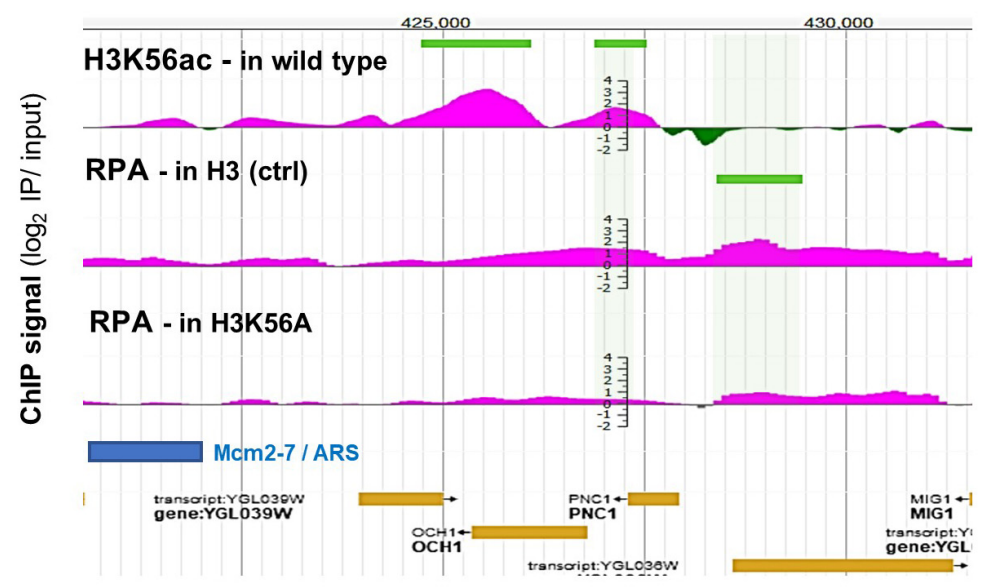

E

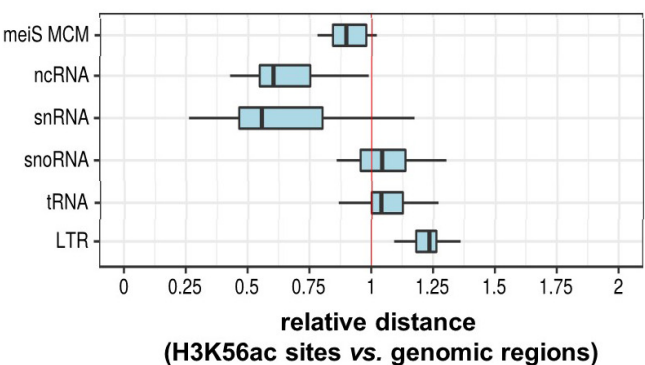

H

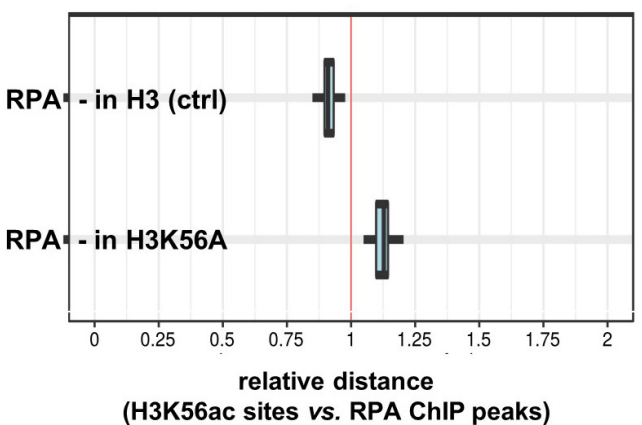

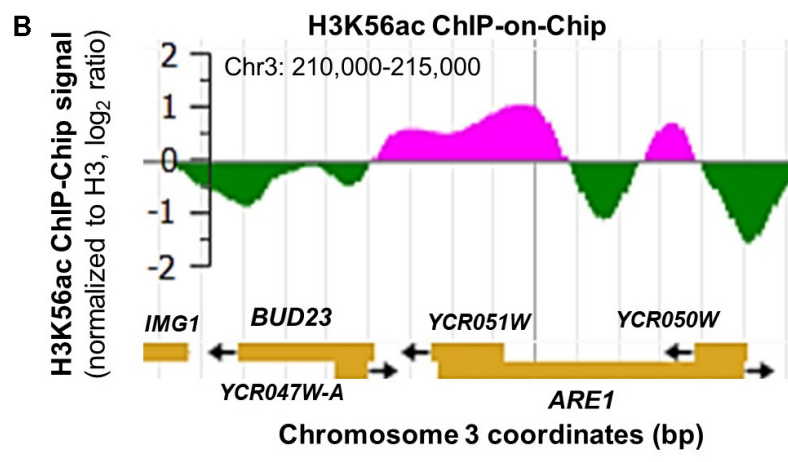

D

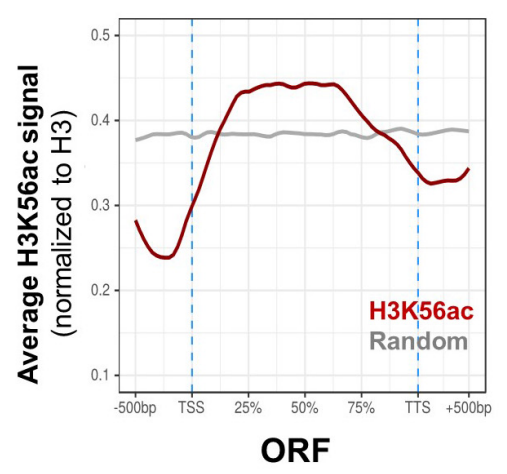

G
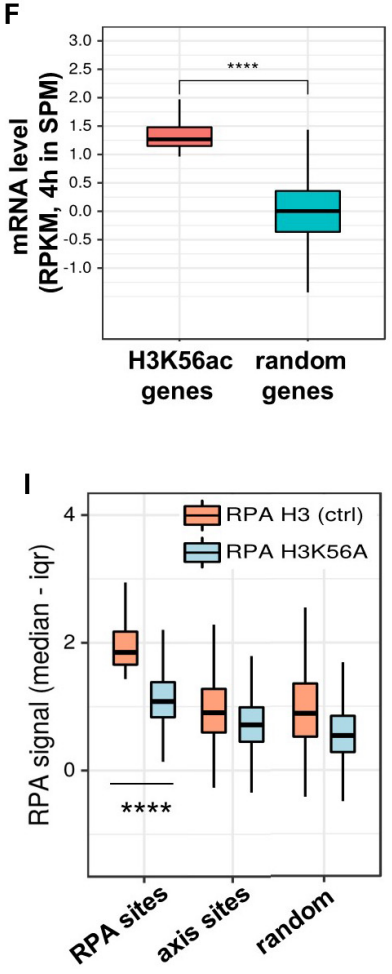

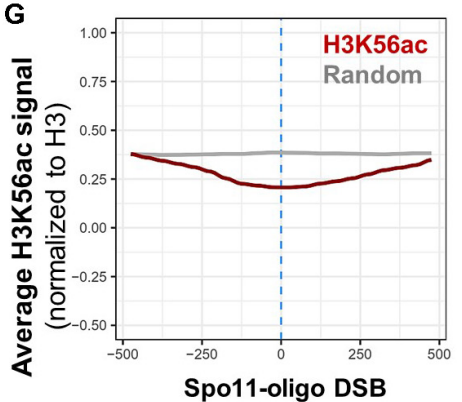

$\mathbf{J}$

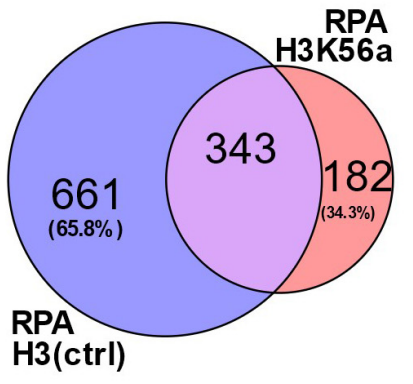




\begin{abstract}
FIGURE 4 | Genome-wide mapping of H3K56ac and RPA. (A) H3K56ac capture using an anti-H3K56ac antibody. H3 (C-ter) ChIP was performed to normalize to H3 occupancy. ChIP-qPCR enrichments were measured at ERG1 and rDNA. Error bars: SEM. (B) H3K56ac signal detected by microarray (at $5 \mathrm{~h}$ in SPM). IP/input ratios were normalized to H3 occupancy (Borde et al., 2009). (C) Genome browser snapshot showing the distribution of H3K56ac in wild type cells and RPA enrichment in $\mathrm{H} 3$ (ctrl) and H3K56A cells. Peaks are highlighted by horizontal green lines. (D) Metagene profile of H3K56ac over protein-coding genes. Red curve shows the median H3K56ac signal. Gray curve: random signal. (E) Relative distance of H3K56ac peaks from various genomic regions. Vertical red line: random distances. Shift toward the left or right sectors: clustering or repulsion between H3K56ac peaks and the studied genomic elements. Mei MCM: meiotic replication origins (Blitzblau et al., 2012). (F) Genes associated with high H3K56ac levels show increased mRNA expression compared to random genes. Statistical significance: $p<0.0001$ (Mann-Whitney $U$ test). RNA-seq data are from Brar et al. (2012). (G) Depletion of H3K56ac over Spo11-oligo DSBs (Mohibullah and Keeney, 2017). Gray line: random signal. (H) Relative distance of H3K56ac peaks from RPA peaks in H3 (ctrl) and H3K56A cells. Vertical red line: random distance. (I) Distribution of RPA ChIP signal at RPA binding sites, axis sites (Mer2; Panizza et al., 2011), and random sites in H3 (ctrl) and H3K56A cells. Statistical significance: $p<0.0001$ (Mann-Whitney $U$ test). (J) Overlap of RPA peaks in H3 (ctrl) and H3K56A cells.
\end{abstract}

DSB, chromosome axis, and repair genes were properly transcribed in H3K56A cells, including SPO11 and RFA1 (Supplementary Figure S1), which were both essential for subsequent meiotic DSB mapping using RPA ChIP (see later). Sporulation efficiency and spore viability of the H3K56A mutant was not different from H3 (ctrl) expressing wild-type H3 (Figure 3D).

Next, we analyzed DSB formation in $\mathrm{H} 3 d m c 1 \Delta$ and H3K56A $d m c 1 \Delta$ cells that accumulate unrepaired DSB ends. DSB frequencies were determined by Southern blot within the natural hotspot region BUD23-ARE1 (Figure 3E). Quantification of DSBs in wild-type and H3 (ctrl) strains confirmed the correct location, timing and frequency of DSBs in plasmid shuffle cells, demonstrating that our system accurately reports DSB formation. Importantly, a threefold reduction of DSB levels was observed in the H3K56A mutant, which was subsequently confirmed by an independent RPA ChIP method capturing Rfa1-covered ssDNA flanking Spo11-oligo DSBs (Figures 3F-H). In plasmid shuffle cells, RPA levels increased in the BUD23 hotspot region by $5 \mathrm{~h}$ in SPM when DSBs are formed, and H3K56A mutants showed a $\sim$ threefold decrease in RPA levels relative to $\mathrm{H} 3$ (ctrl) (Figure 3G), consistent with our Southern blot results (Figure 3E). In addition, the RPA profiles of H3 (ctrl) and wild-type cells (Borde et al., 2009) were positively correlated (Figure $3 \mathbf{H}$ ), whereas the RPA binding sites did not correlate with the binding of $\mathrm{Mcm} 2-7$ replicative helicase that marks meiotic DNA replication (Supplementary Figure S2). These results collectively demonstrate that RPA enrichment is an adequate indicator of meiotic DSB locations and frequencies.

Notably, the BUD23 and ERG1 hotspot regions were flanked by H3K56ac at the time of DSB formation as measured by H3K56ac ChIP-qPCR and ChIP-chip (Figures 4A,B). We note that the H3K56ac ChIP signal was undetectable in asf $1 \Delta$ cells that are deficient in H3K56 acetylation (Figure 1A), confirming the specificity of our ChIP assay. Furthermore, the H3K56ac signal was also depleted in wild-type cells within the recombinationally cold $r D N A$ region (Figure $4 \mathrm{~A}$ ). The same associations were observed at the genomic scale (Figure 4C and JBrowse link), suggesting that the presence of H3K56 residue and/or deposition of H3K56-acetylated nucleosomes near DSB hotspots is required for complete DSB formation. Our H3K56ac ChIP results show that H3K56 acetylation is preferentially associated with protein-coding ORFs (Figure 4D) and is depleted from promoters and transcription termination sites [in line with published ChIP-qPCR data in mitotic cells (Schneider et al., 2006)]. The relative distance of H3K56ac from several genomic elements showed a clear non-random distribution (Figure 4E) as H3K56ac peaks were preferentially associated with Mcm2-7 helicase binding sites (Blitzblau et al., 2012), ncRNA and snRNA genes, but were further away from snoRNAs, tRNAs, and LTR retrotransposons compared to a computer-randomized chromosomal distribution (vertical red line in the figure). The proximity of H3K56ac peaks to Mcm2-7 binding sites agrees with the suggested role of H3K56 acetylation in marking nascent (replicating) chromatin upon replicative and repair DNA synthesis (Yu et al., 2012). Regarding meiotic gene expression (Figure $4 \mathbf{F}$ ), protein-coding genes enriched in H3K56ac showed significantly higher mRNA expression levels in meiosis (at $4 \mathrm{~h}$ in SPM) than random genes [ $p<0.0001$; RNA-seq data are from Brar et al. (2012)]. Increased expression of H3K56-acetylated genes supports previous results in mitotic cells (Schneider et al., 2006; Williams et al., 2008), indicating that $\mathrm{H} 3 \mathrm{~K} 56 \mathrm{ac}$ is an active chromatin mark associated with transcription.

Regarding meiotic DSB sites, chromosomal distribution of the H3K56ac signal showed a depletion over Spo11-oligo DSBs (Figure 4G), which precisely mark recombination hotspots. However, genomic positions of H3K56-acetylated histones were preferentially enriched adjacent to the identified RPA binding sites relative to random distance (Figure $4 \mathrm{H}$, RPA H3 ctrl). Importantly, the RPA ChIP signal detected in the H3K56A mutant was strongly reduced at the RPA sites identified in the H3 (ctrl) strain $(p<0.0001$, Mann-Whitney $U$ test), as opposed to chromosome axis sites (Mer2-tagged; Panizza et al., 2011) and randomly selected sites that did not differ between control and mutant strains (Figure 4I). The preferential decrease of RPA ChIP signal at RPA binding sites in the H3K56A mutant demonstrates the specificity of H3K56A substitution mutation for recombination hotspot regions, accumulating hyper-resected ssDNA in the vicinity of DSBs. The specific decrease in the number of RPA peaks in the H3K56A mutant is highlighted in Figure 4J. This Venn diagram analysis shows that $65.8 \%$ of RPA peaks were eliminated by the H3K56A mutation (661 sites out of 1004), whereas 343 RPA sites were not affected or the signal was even increased (182 peaks). The latter RPA binding sites may represent unscheduled DSBs that are not related to H3K56 acetylation, which is clearly apparent from the increased relative distance between $\mathrm{H} 3 \mathrm{~K} 56 \mathrm{ac}$ histones and RPA ChIP peaks detected in the H3K56A mutant (Figure 4H, RPA-H3K56A). 


\section{DISCUSSION}

The above functional results highlight the association of $\mathrm{H} 3 \mathrm{~K} 56$ acetylation and meiotic DSB formation, suggesting that H3K56acetylated histones are required to produce normal levels of DSBs within recombination hotspot regions. Nevertheless, the exact molecular mechanism underlying the DSB-promoting effect of H3K56 acetylation has yet to be clarified. The cause of the observed differences between the two mutant systems (histone modifying enzyme deletion vs. histone mutation) is currently not known. We obtained complementary results that are fully consistent with recent data identifying differences in the RNAPII binding profile of $r t t 109 \Delta$ and H3K56R mutants (Topal et al., 2019). Based on the genome-scale analysis of H3K56A cells, we propose that lack of H3K56 acetylation affects the stability or turnover rate of the well-positioned first nucleosomes flanking DSB sites (consistent with Kaplan et al. (2008)], and this may reduce the efficiency of Spo11catalyzed DNA cleavage. Moreover, the absence of H3K56ac mark could reduce the rate of DSB end dissociation from nucleosomes flanking DSB sites, impeding the timely resection and processing of DSB ends. Alternatively, the H3K56A mutation may exert its effect indirectly on DSB formation, however, this is probably independent of changes in transcription since no differential gene expression was detected in the H3K56A mutant. A further possibility could be that H3K56 acetylation promotes the interaction of $\mathrm{H} 3 \mathrm{~K} 4 \mathrm{me} 3$, Spp1, and Mer2 during the loop tethering process. The recently identified epistatic relationship of $\mathrm{H} 3 \mathrm{~K} 56 \mathrm{ac}$ and $\mathrm{H} 3 \mathrm{~K} 4 \mathrm{me} 3$ supports this hypothesis and seems particularly important in this regard (Voichek et al., 2018). H3K56ac was found to act upstream of Set1C and H3K4 methylation, generating complementary $\mathrm{H} 3 \mathrm{~K} 56 \mathrm{ac} / \mathrm{H} 3 \mathrm{~K} 4 \mathrm{me} 3$ histone modification patterns along newly replicated chromatin. These functional relationships could readily allow close cooperation between $\mathrm{H} 3 \mathrm{~K} 56 \mathrm{ac}$ and $\mathrm{H} 3 \mathrm{~K} 4 \mathrm{me} 3$ during meiotic recombination, especially because newly replicated chromatin is rich in H3K56ac and DNA replication is mechanically coupled to meiotic DSB formation (Murakami and Keeney, 2014). We assume that these complex spatial interactions occur in the

\section{REFERENCES}

Abshiru, N., Ippersiel, K., Tang, Y., Yuan, H., Marmorstein, R., Verreault, A., et al. (2013). Chaperone-mediated acetylation of histones by Rtt109 identified by quantitative proteomics. J. Proteomics 81, 80-90. doi: 10.1016/j.jprot.2012. 09.026

Acquaviva, L., Székvölgyi, L., Dichtl, B., Dichtl, S. B., de La Roche Saint André, C., Nicolas, A., et al. (2013). The COMPASS subunit Spp1 links histone methylation to initiation of meiotic recombination. Science 339, 215-218. doi: 10.1126/ science. 1225739

Baudat, F., Buard, J., Grey, C., Fledel-Alon, A., Ober, C., Przeworski, M., et al. (2010). PRDM9 is a major determinant of meiotic recombination hotspots in humans and mice. Science 327, 836-840. doi: 10.1126/science.1183439

Biswas, M., Voltz, K., Smith, J. C., and Langowski, J. (2011). Role of histone tails in structural stability of the nucleosome. PLoS Comput. Biol. 7: e1002279. doi: 10.1371/journal.pcbi.1002279

Blitzblau, H. G., Chan, C. S., Hochwagen, A., and Bell, S. P. (2012). Separation of DNA replication from the assembly of break-competent meiotic chromosomes. PLoS Genet. 8:e1002643. doi: 10.1371/journal.pgen.1002643 context of 3D chromatin structure. This could be detected by chromosome conformation capture methods (Dekker et al., 2017). Future use of these C-based approaches, together with mutant analyses, is expected to provide a deeper understanding of meiotic DSB formation with regards to the role of H3K4 methylation, H3K56 acetylation, and other potentially relevant histone modifications.

\section{DATA AVAILABILITY STATEMENT}

Datasets generated for this study can be accessed in Supplementary Table S1 and via JBrowse (login: h3k56ac, password: Mozaic4, http://geneart.med.unideb.hu/pub/ h3k56ac). Raw data are availabe at GEO (GSE37487).

\section{AUTHOR CONTRIBUTIONS}

ZK and LH analyzed the genomic data. LS performed the experiments and wrote the manuscript.

\section{FUNDING}

LS received funding from HAS-Lendület-LP2015-9/2015, NKFIH-NNE-130913, and GINOP-2.3.2-15-2016-00024.

\section{ACKNOWLEDGMENTS}

The authors thank Alain Nicolas (Institut Curie) for the conceptual support and critical comments.

\section{SUPPLEMENTARY MATERIAL}

The Supplementary Material for this article can be found online at: https://www.frontiersin.org/articles/10.3389/fcell.2019.00364/ full\#supplementary-material

Borde, V., Robine, N., Lin, W., Bonfils, S., Géli, V., and Nicolas, A. (2009). Histone $\mathrm{H} 3$ lysine 4 trimethylation marks meiotic recombination initiation sites. EMBO J. 28, 99-111. doi: 10.1038/emboj.2008.257

Brar, G. A., Yassour, M., Friedman, N., Regev, A., Ingolia, N. T., and Weissman, J. S. (2012). High-resolution view of the yeast meiotic program revealed by ribosome profiling. Science 335, 552-557. doi: 10.1126/science.1215110

Buning, R., and Van Noort, J. (2010). Single-pair FRET experiments on nucleosome conformational dynamics. Biochimie 92, 1729-1740. doi: 10.1016/j.biochi.2010. 08.010

Celic, I., Masumoto, H., Griffith, W. P., Meluh, P., Cotter, R. J., Boeke, J. D., et al. (2006). The Sirtuins Hst3 and Hst4p preserve genome integrity by controlling Histone H3 Lysine 56 Deacetylation. Curr. Biol. 16, 1280-1289. doi: 10.1016/j. cub.2006.06.023

Dekker, J., Belmont, A. S., Guttman, M., Leshyk, V. O., Lis, J. T., Lomvardas, S., et al. (2017). The 4D nucleome project. Nature 549, 219-226. doi: 10.1038/ nature23884

Govin, J., Dorsey, J., Gaucher, J., Rousseaux, S., Khochbin, S., and Berger, S. L. (2010). Systematic screen reveals new functional dynamics of histones H3 and H4 during gametogenesis. Genes Dev. 24, 1772-1786. doi: 10.1101/gad.1954910 
Kaplan, T., Liu, C. L., Erkmann, J. A., Holik, J., Grunstein, M., Kaufman, P. D., et al. (2008). Cell cycle- and chaperone-mediated regulation of $\mathrm{H} 3 \mathrm{~K} 56 \mathrm{ac}$ incorporation in yeast. PLoS Genet. 4:e1000270. doi: 10.1371/journal.pgen. 1000270

Karányi, Z., Halász, L., Acquaviva, L., Jónás, D., Hetey, S., Boros-Oláh, B., et al. (2018). Nuclear dynamics of the Set1C subunit Spp1 prepares meiotic recombination sites for break formation. J Cell Biol. 217, 3398-3415. doi: 10. 1083/jcb.201712122

Keeney, S., Giroux, C. N., and Kleckner, N. (1997). Meiosis-specific DNA doublestrand breaks are catalyzed by Spo11, a member of a widely conserved protein family. Cell 88, 375-384. doi: 10.1016/s0092-8674(00)81876-0

Luger, K., Rechsteiner, T., Flaus, A., Waye, M., and Richmond, T. J. (1997). Characterization of nucleosome core particles containing histone proteins made in bacteria. J. Mol. Biol. 272, 301-311. doi: 10.1006/jmbi.1997.1235

Messiaen, S., Guiard, J., Aigueperse, C., Fliniaux, I., Tourpin, S., Barroca, V., et al. (2016). Loss of the histone chaperone ASF1B reduces female reproductive capacity in mice. Reproduction 151, 477-489. doi: 10.1530/REP-15-0327

Mohibullah, N., and Keeney, S. (2017). Numerical and spatial patterning of yeast meiotic DNA breaks by Tel1. Genome Res. 27, 278-288. doi: $10.1101 /$ gr.213587.116

Murakami, H., and Keeney, S. (2014). Temporospatial coordination of meiotic dna replication and recombination via DDK recruitment to replisomes. Cell 158, 861-873. doi: 10.1016/j.cell.2014.06.028

Pan, J., Sasaki, M., Kniewel, R., Murakami, H., Blitzblau, H. G., Tischfield, S. E., et al. (2011). A hierarchical combination of factors shapes the genome-wide topography of yeast meiotic recombination initiation. Cell 144, 719-731. doi: 10.1016/j.cell.2011.02.009

Panizza, S., Mendoza, M. A., Berlinger, M., Huang, L., Nicolas, A., Shirahige, K., et al. (2011). Spol1-accessory proteins link double-strand break sites to the chromosome axis in early meiotic recombination. Cell 146, 372-383. doi: 10.1016/j.cell.2011.07.003

Parpanov, E. D., Petkov, P. M., and Paigen, K. (2010). Prdm9 controls activation of mammalian recombination hotspots. Science 327:835. doi: 10.1126/science. 1181495

Recht, J., Tsubota, T., Tanny, J. C., Diaz, R. L., Berger, J. M., Zhang, X., et al. (2006). Histone chaperone Asf1 is required for histone H3 lysine 56 acetylation, a modification associated with $\mathrm{S}$ phase in mitosis and meiosis. Proc. Natl. Acad. Sci. U.S.A. 103, 6988-6993. doi: 10.1073/pnas.0601676103

Rufiange, A., Jacques, P. E., Bhat, W., Robert, F., and Nourani, A. (2007). Genomewide replication-independent histone $\mathrm{H} 3$ exchange occurs predominantly at promoters and implicates H3 K56 acetylation and Asf1. Mol. Cell 27, 393-405. doi: 10.1016/j.molcel.2007.07.011

Schneider, J., Bajwa, P., Johnson, F. C., Bhaumik, S. R., and Shilatifard, A. (2006). Rtt109 is required for proper H3K56 acetylation: a chromatin mark associated with the elongating RNA polymerase II. J. Biol. Chem. 281, 37270-37274. doi: 10.1074/jbc.C600265200

Simon, M., North, J. A., Shimko, J. C., Forties, R. A., Ferdinand, M. B., Manohar, M., et al. (2011). Histone fold modifications control nucleosome unwrapping and disassembly. Proc. Natl. Acad. Sci. U.S.A. 108, 12711-12716. doi: 10.1073/ pnas. 1106264108

Simoneau, A., Delgoshaie, N., Celic, I., Dai, J., Abshiru, N., Costantino, S., et al. (2015). Interplay between histone H3 lysine 56 deacetylation and chromatin modifiers in response to DNA damage. Genetics 200, 185-205. doi: 10.1534/ genetics.115.175919

Sommermeyer, V., Beneut, C., Chaplais, E., Serrentino, M. E., and Borde, V. (2013). Spp1, a member of the Set1 Complex, promotes meiotic DSB formation in promoters by tethering histone H3K4 methylation sites to chromosome axes. Mol. Cell 49, 43-54. doi: 10.1016/j.molcel.2012.11.008

Szekvolgyi, L., and Nicolas, A. (2010). From meiosis to postmeiotic events: homologous recombination is obligatory but flexible. FEBS J. 277, 571-589. doi: 10.1111/j.1742-4658.2009.07502.x

Székvölgyi, L., and Nicolas, A. (2010). From meiosis to postmeiotic events: homologous recombination is obligatory but flexible. FEBS J. 277, 571-589. doi: 10.1111/j.1742-4658.2009.07502.x

Székvölgyi, L., Ohta, K., and Nicolas, A. (2015). Initiation of meiotic homologous recombination: flexibility, Impact of Histone modifications, and chromatin remodelling. Cold Spring Harb. Perspect. Biol. 7, 447-462. doi: 10.1101/ cshperspect.a016527

Tian, H., Billings, T., and Petkov, P. M. (2018). CXXC1 is redundant for normal DNA double-strand break formation and meiotic recombination in mouse. PLoS Genet. 14:e1007657. doi: 10.1371/journal.pgen.1007657

Topal, S., Vasseur, P., Radman-Livaja, M., and Peterson, C. L. (2019). Distinct transcriptional roles for Histone H3-K56 acetylation during the cell cycle in Yeast. Nat. Commun. 10, 1-13. doi: 10.1038/s41467-019-12400-5

Tsubota, T., Berndsen, C. E., Erkmann, J. A., Smith, C. L., Yang, L., Freitas, M. A., et al. (2007). Histone H3-K56 acetylation is catalyzed by histone chaperonedependent complexes. Mol. Cell 25, 703-712. doi: 10.1016/j.molcel.2007.02. 006

Voichek, Y., Mittelman, K., Gordon, Y., Bar-Ziv, R., Lifshitz Smit, D., Shenhav, R., et al. (2018). Epigenetic control of expression homeostasis during replication is stabilized by the replication checkpoint. Mol. Cell 70, 1121.e9-1133.e9. doi: 10.1016/j.molcel.2018.05.015

Watanabe, S., Radman-Livaja, M., Rando, O. J., and Peterson, C. L. (2013). A histone acetylation switch regulates H2A.Z deposition by the SWRC remodeling enzyme. Science 340, 195-199. doi: 10.1126/science.122 9758

White, C. L., Suto, R. K., and Luger, K. (2001). Structure of the yeast nucleosome core particle reveals fundamental changes in internucleosome interactions. EMBO J. 20, 5207-5218. doi: 10.1093/emboj/20.18.5207

Williams, S. K., Truong, D., and Tyler, J. K. (2008). Acetylation in the globular core of histone $\mathrm{H} 3$ on lysine-56 promotes chromatin disassembly during transcriptional activation. Proc. Natl. Acad. Sci. U.S.A. 105, 9000-9005. doi: 10.1073/pnas.0800057105

Xu, L., and Kleckner, N. (1995). Sequence non-specific double-strand breaks and interhomolog interactions prior to double-strand break formation a meiotic recombination hot spot in yeast. EMBO J. 14, 5115-5128. doi: 10.1002/j.14602075.1995.tb00194.x

Yu, Y., Song, C., Zhang, Q., DiMaggio, P. A., Garcia, B. A., York, A., et al. (2012). Histone H3 lysine 56 methylation regulates DNA replication through its interaction with PCNA. Mol. Cell 46, 7-17. doi: 10.1016/j.molcel.2012. 01.019

Conflict of Interest: The authors declare that the research was conducted in the absence of any commercial or financial relationships that could be construed as a potential conflict of interest.

Copyright (c) 2020 Karányi, Hornyák and Székvölgyi. This is an open-access article distributed under the terms of the Creative Commons Attribution License (CC BY). The use, distribution or reproduction in other forums is permitted, provided the original author(s) and the copyright owner(s) are credited and that the original publication in this journal is cited, in accordance with accepted academic practice. No use, distribution or reproduction is permitted which does not comply with these terms. 